\title{
Using proteomic analysis to investigate uniconazole-induced phytohormone variation and starch accumulation in duckweed (Landoltia punctata)
}

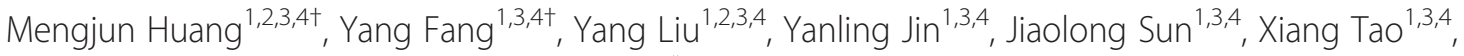 \\ Xinrong $\mathrm{Ma}^{1,3,4}$, Kaize He $\mathrm{H}^{1,3,4}$ and Hai Zhao ${ }^{1,3,4^{*}}$
}

\begin{abstract}
Background: Duckweed (Landoltia punctata) has the potential to remediate wastewater and accumulate enormous amounts of starch for bioethanol production. Using systematical screening, we determined that the highest biomass and starch percentage of duckweed was obtained after uniconazole application. Uniconazole contributes to starch accumulation of duckweed, but the molecular mechanism is still unclear.

Results: To elucidate the mechanisms of high starch accumulation, in the study, the responses of L. punctata to uniconazole were investigated using a quantitative proteomic approach combined with physiological and biochemical analysis. A total of 3327 proteins were identified. Among these identified proteins, a large number of enzymes involved in endogenous hormone synthetic and starch metabolic pathways were affected. Notably, most of the enzymes involved in abscisic acid (ABA) biosynthesis showed up-regulated expression, which was consistent with the content variation. The increased endogenous ABA may up-regulate expression of ADP-glucose pyrophosphorylase to promote starch biosynthesis. Importantly, the expression levels of several key enzymes in the starch biosynthetic pathway were up-regulated, which supported the enzymatic assay results and may explain why there is increased starch accumulation.

Conclusions: These generated data linked uniconazole with changes in expression of enzymes involved in hormone biosynthesis and starch metabolic pathways and elucidated the effect of hormones on starch accumulation. Thus, this study not only provided insights into the molecular mechanisms of uniconazole-induced hormone variation and starch accumulation but also highlighted the potential for duckweed to be feedstock for biofuel as well as for sewage treatment.
\end{abstract}

\section{Background}

Renewable biofuels, such as bioethanol, derived from biomass is considered to be the most promising alternative to petroleum liquid fuels. The development of bioethanol can reduce greenhouse gas emissions and meet the strong global demand for energy [1]. Current commercial production

\footnotetext{
* Correspondence: zhaohai@cib.ac.cn

${ }^{\dagger}$ Equal contributors

'Chengdu Institute of Biology, Chinese Academy of Sciences, Chengdu 610041, China

${ }^{3}$ Key Laboratory of Environmental and Applied Microbiology, Chinese Academy of Sciences, Chengdu 610041, China

Full list of author information is available at the end of the article
}

of ethanol fuel has focused on using starch and sugar from maize, sugarcane [2], cassava [3], and sweet potato [4]. However, there is some controversy surrounding these feedstocks that often compete with food crops for arable land $[5,6]$. For the most abundant lignocellulosic sources, there is still a lack of an economical, efficient, and environmentally beneficial pretreatment process for ethanol biofuel production [7]. Therefore, exploration and research of novel alternative resources for bioethanol production has attracted tremendous interest.

Duckweed, classified as a separate family Lemnaceae earlier, arose from the arum or aroid family [8] and therefore, often are classified as the subfamily Lemnoideae 
within the Araceae. As a global adaptable aquatic macrophyte [9], the plant is a potential alternative feedstock for bioethanol production [10-14] because of its high starch accumulation and very low lignin percentage [15]. Duckweed has been used for wastewater treatment such as domestic sewage and industrial wastewater; the biomass generated was also sufficient for biofuels production while removing nutrients $[16,15,17,18]$. The relative growth rate of duckweed is $12 \mathrm{~g} / \mathrm{m}^{2} /$ day dry weight (DW) in warm regions [19]; the average annual rate is $7.26 \mathrm{~g} \mathrm{DW} / \mathrm{m}^{2} /$ day, enough to yield 26.50 tons per hectare, indicating that duckweed has a higher yield than most terrestrial plants [20]. By manipulating the growth conditions, such as $\mathrm{pH}$, phosphate concentration, nutrient starvation, and light conditions, duckweed can accumulate starch up to $52.9 \%$ of its DW [10,12, 21, 22]. Because of these key attributes for a potential biofuel feedstock, duckweed has received much attention from academia, industry, and the government. At present, there is some pilot-scale research on duckweed and its applications [23, 24].

Plant growth regulators (PGRs) have been widely used to manipulate plant growth and the yield of crops $[25,26]$. To enhance the biomass and starch accumulation of duckweed for bioethanol fermentation under large-scale cultivation, we also used PGRs to regulate duckweed growth. Approximately twenty PGRs were screened systematically at different concentration gradients. One of the main findings was that uniconazole applied as spraying the surface of a population at $800 \mathrm{mg} / \mathrm{L}$ yielded the highest biomass and starch content of duckweed. Uniconazole, an active member of the triazole family, has been used in agriculture to enhance stress tolerance $[27,28]$ and grain quality of rice [29] and wheat [30] by inhibition of gibberellin (GA) biosynthesis [25]. Similarly, it has significantly increased the starch content of potato tubers by approximately $8 \%$ [31] and starch grain accumulation in lotus rhizomes [32]. Plants respond to uniconazole through various biochemical and physiological processes, but the molecular mechanism is still unclear. Whether or not the uniconazole-induced starch accumulation mainly depends on endogenous GA, is still unknown. There has thus far been little research linking uniconazole with expression changes in endogenous hormone biosynthesis enzymes and on the roles of certain hormone variations that cause high starch accumulation.

Bioinformatics, such as transcriptomics and proteomics, are useful as they could provide global information about gene expression. These methods could be powerful to analyze the metabolic pathways of non-model plants, since it is difficult to elucidate the metabolic mechanism without genetic transformation system. The protein, as the functional executor, is most closely related to physiological changes. Hence, it is more important to investigate downstream protein expression by proteomic technology. Our previous proteomic study of L. punctata has reported its high starch accumulation and low lignin percentage under nutrient starvation [33]. However, how duckweed accumulates high levels of starch with uniconazole application is still unclear. In the present study, the proteomic analysis of the uniconazole-treated duckweed was performed with isobaric tags for the relative and absolute quantification (iTRAQ) technique [34]. These results provide important information about the molecular mechanisms of hormone changes and starch accumulation with uniconazole application, which can further develop duckweed as a bioenergy crop.

\section{Results and discussion}

\section{Proteomic research of $L$. punctata after application of uniconazole}

The establishment of a $L$. punctata database based on transcriptome data contributed to protein identification. In our study, a total of 369,230 spectra were obtained from iTRAQ liquid chromatography-tandem mass spectrometry (LC-MS/MS) proteomic analysis. Because the whole genome of L. punctata has not yet been completely identified, a protein sequence database was established using the mRNA transcripts derived from our RNA-Seq data of $L$. punctata under uniconazole treatment. The translated mRNA sequence database was generated by translation of all open reading frames (ORFs) for these transcripts by 6-frame translation [35]. After searching against the database, 40,996 unique spectra that met strict identification criteria were matched to 13,457 unique peptides and 3327 proteins.

In terms of protein molecular weight distribution, there was very good coverage (all proteins) for a wide range for proteins larger than $20 \mathrm{kDa}$ (Fig. 1a). In addition, most of the proteins were identified with good sequence coverage; approximately $66 \%$ of the proteins were with more than $5 \%$ of the sequence coverage, and approximately $44 \%$ were with $10 \%$ of the sequence coverage (Fig. 1b). All identified unique proteins were classified into three ontologies: biological process, cellular component, or molecular function (Fig. 1c). The main subcategories within the cellular component proteins were cell $(23.52 \%)$ and cell part (23.52 \%). In the molecular function category, the most frequently detected gene ontology (GO) terms included catalytic activity and binding, representing 47.86 and $40.22 \%$, respectively. The biological process category was mainly represented by metabolic process and cellular process proteins, representing 17.62 and $17.09 \%$, respectively. Using a cutoff of a fold change $>1.2$ or $<0.8$ with a $p$-value of less than $0.05,969$ proteins were quantified as having significant changes in expression. There were 578 proteins that had increased abundance (Additional file 1) and 391 that had decreased 


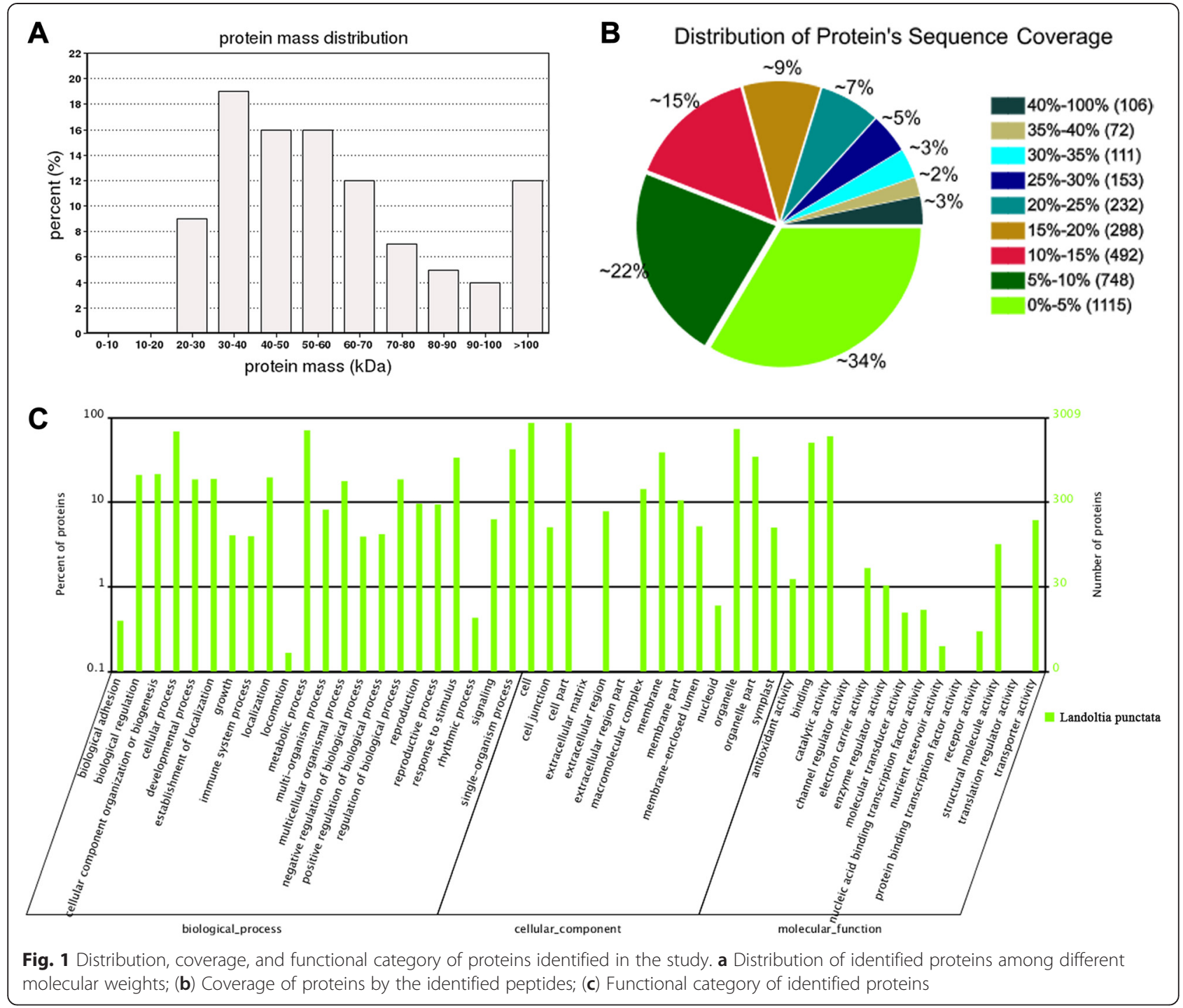

(Additional file 2) after uniconazole application. The proteins that had differential expression among different time points are listed in the Table 1. Among these proteins, 420 proteins were found to be responsive to uniconazole between 240 and $0 \mathrm{~h}$ (Table 1).

Metabolic pathway enrichment analysis was performed for the differentially displayed proteins to determine the affected cellular metabolism. These proteins were matched to the proteins annotated with the KEGG pathway database, and the frequencies of the responsive proteins in each KEGG pathway were compared to determine statistical authenticity of the involvement of that pathway induced by uniconazole. At 2, 5, 72, and $240 \mathrm{~h}$ after spraying uniconazole, 21, 12, 65, and 94 KEGG pathways were affected, respectively, with $p$-values less than 0.05 . Carotenoid biosynthesis (KO00906) and starch and sucrose metabolism (KO00500) were also enriched (Additional file 3), suggesting that these proteins in these pathways were points

\begin{tabular}{llll}
\hline Comparisons & $\begin{array}{l}\text { Up-regulated } \\
\text { proteins (No.) }\end{array}$ & $\begin{array}{l}\text { Down-regulated } \\
\text { proteins (No.) }\end{array}$ & Total (No.) \\
\hline 2 h vs 0 h & 17 & 9 & 26 \\
5 h vs 0 h & 8 & 7 & 15 \\
72 h vs 0 h & 142 & 75 & 217 \\
240 h vs 0 h & 273 & 147 & 420 \\
5 h vs 2 h & 8 & 26 & 34 \\
72 h vs 2 h & 117 & 96 & 213 \\
240 h vs 2 h & 221 & 162 & 383 \\
72 h vs 5 h & 130 & 66 & 196 \\
240 h vs 5 h & 236 & 146 & 382 \\
240 h vs 72 h & 104 & 50 & 154 \\
\hline
\end{tabular}

Numbers of proteins that had differential expression were listed in the table 
highly expressed and may be active during the treatment condition. These pathways are very important for hormone variation and starch accumulation, which are our main focuses. Hence, the expression profiles of these enzymes involved in relevant pathways need more investigation.

The establishment of a protein sequence database based on transcriptomic data is suitable for proteomic analysis of species that have not yet been whole-genome sequenced. There were also successful cases in Bugula neritina [36], Macleaya cordata, and Macleaya microcarpa [37], and L. punctata under nutrient starvation from our previous proteomic study [33]. There was a high concordance in variation trends of enzymes when comparing protein with transcript in response to uniconazole. On the one hand, most of the identified enzymes involved in hormone biosynthesis, such as zeaxanthin epoxidase (EC: 1.14.13.90; ZEP) and 9-cis-epoxycarotenoid dioxygenase (EC: 1.13.11.51; NCED) (Additional file 1), were consistent with the transcripts in terms of trends. The upregulated expression of these enzymes contributed to ABA biosynthesis. On the other hand, many key enzymes in the starch metabolic pathway, such as ADP-glucose pyrophosphorylase (EC: 2.7.7.27; AGP) and granule bound starch synthase (EC: 2.4.1.11; GBSS) shared the same upregulated expression direction at both the protein and transcript levels. Their expression contributes to rapid accumulation of high levels of starch. However, some enzymes displayed different trends between proteome and transcriptome. For example, the transcript expression of soluble starch synthetase (EC: 2.4.1.21; SSS) decreased, but the protein expression displayed no significant change. Several reasons may account for this variation. First, there are various expression orders and abundance changes at different phases. These processes may also include posttranscriptional, translational and/or posttranslational regulation [38]. Second, the shortage of genome information also affects identification of some unique proteins. Moreover, existing techniques have limitations in identification and quantification at the two levels. A similar conclusion was also obtained by our earlier study with transcriptomics [39] and proteomics data [33] collected from $L$. punctata under nutrient starvation. Importantly, our time-series proteomic results can provide a more direct expression pattern than other methods, which also supported the physiological changes after application of uniconazole.

\section{Phytohormone change of $L$. punctata under uniconazole treatment}

The contents of abscisic acid (ABA) and zeatin-riboside $(\mathrm{ZR})$ increased, but the gibberellin (GA) content decreased with uniconazole application. ABA had the highest content among these measured phytohormones of all samples. There was a significant increase in ABA content from 61.47 to $166.53 \mathrm{ng} / \mathrm{g}$ FW at $240 \mathrm{~h}$ (Fig. 2). Similarly, ZR, a type of cytokinin, also slightly increased from 7.73 to11.87 ng/g FW by $240 \mathrm{~h}$. On the contrary, $\mathrm{GA}_{1+3}$ content decreased from 9.25 to $5.57 \mathrm{ng} / \mathrm{g} \mathrm{FW}$ at $240 \mathrm{~h}$ while $\mathrm{GA}_{4+7}$ content maintained a stable low level.

The expression levels of related enzymes involved in hormone synthetic pathways were also analyzed. The biosynthesis of ABA, ZR, and GA were all from mevalonate, which generates from terpenoid backbone biosynthesis (Fig. 3). The expression levels of most of the enzymes associated with the ABA biosynthetic pathway were up-regulated. For example, 9-cis-epoxycarotenoid dioxygenase (EC: 1.13.11.51; NCED), the rate-limiting enzyme that catalyzes the synthesis of xanthoxin by cleaving 9'-cis'-neoxanthinc and 9-cis-violaxanthin [40], increased 1.91-fold at $2 \mathrm{~h}$ (Fig. 3). Beta-glucosidase (EC: 3.2.1.21; GBA3), regulating the final conversion of ABAglucose ester into bioactive ABA [41], increased 1.58fold at $240 \mathrm{~h}$. Zeaxanthin epoxidase (EC: 1.14.13.90; ZEP) and xanthoxin dehydrogenase (EC: 1.1.1.288; ABA2) also increased 2.80- and 1.95-fold at $240 \mathrm{~h}$, respectively. Other enzymes, such as abscisic-aldehyde oxidase (EC: 1.2.3.14; AAO3), neoxanthin synthase (EC: 5.3.99.9; NSY), and abscisate beta-glucosyltransferase (EC: 2.4.1.263; AOG), displayed no significant change or were unidentified. Moreover, all enzymes related to the biosynthesis of GA and ZR had no differential expression or was unidentified. Gibberellin-44 dioxygenase (EC: 1.14.11.12; G44OX) and gibberellin 3-beta-dioxygenase (EC: 1.14.11.15; GA3OX) that participate in GA biosynthesis and cytokinin trans-hydroxylase (CYP735A) involved in ZR biosynthesis showed no differential change. Other key enzymes, such as ent-copalyl diphosphate synthase (EC: 5.5.1.13; CPS), ent-kaurene synthase (EC: $4.2 .3 .19 ; \mathrm{KS}$ ), and adenylate isopentenyltransferase (EC: 2.5.1.27; IPT) were unidentified.

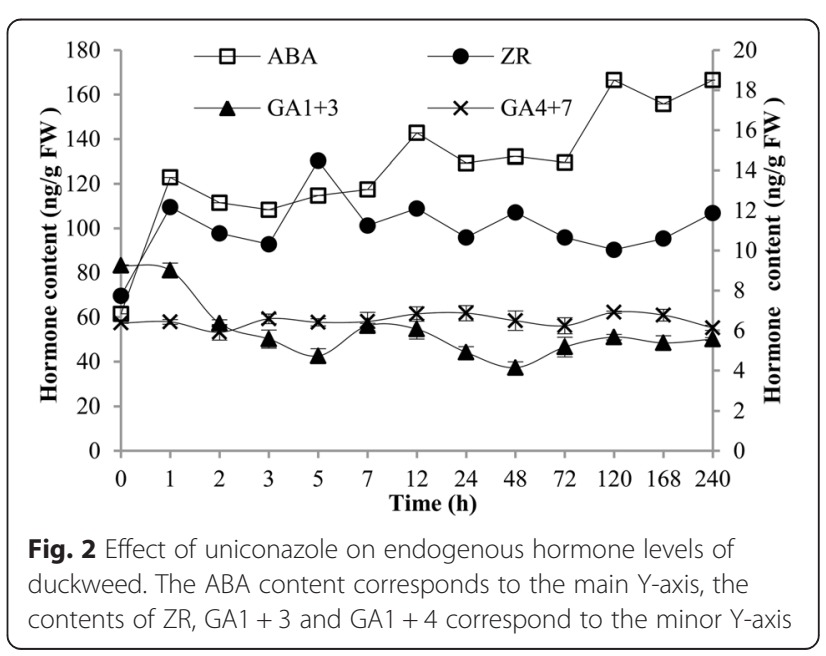




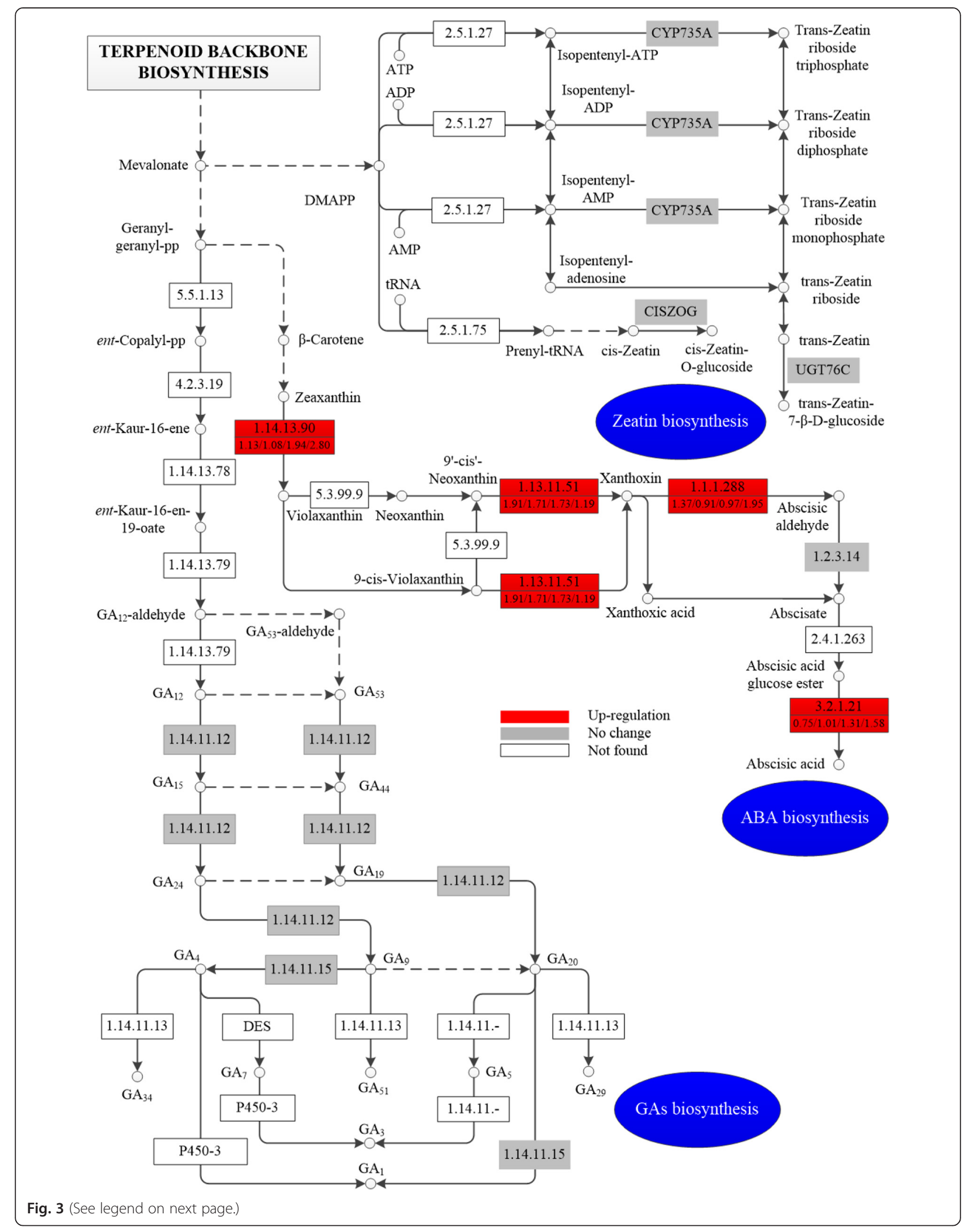


(See figure on previous page.)

Fig. 3 Expression patterns of some enzymes involved in hormones biosynthesis. Red boxes indicate the up-regulated enzymes in response to uniconazole, gray means no significant difference was observed, and white means this enzyme was not found in this study. The numbers in the upper half of the boxes correspond to EC numbers, and the numbers in the lower half correspond to the ratios of expression levels of these enzymes at 2, 5, 72, and 240 h compared with the control levels. 5.5.1.13: ent-copalyl diphosphate synthase; 4.2.3.19: ent-kaurene synthase; 1.14.13.78: ent-kaurene oxidase; 1.14.13.79: ent-kaurenoic acid oxidase; 1.14.11.12: gibberellin-44 dioxygenase; 1.14.11.15: gibberellin 3beta-dioxygenase; 1.14.11.13: gibberellin 2beta-dioxygenase; 1.14.13.90: zeaxanthin epoxidase; 5.3.99.9: neoxanthin synthase; 1.13.11.51: 9-cis-epoxycarotenoid dioxygenase; 1.1.1.288: xanthoxin dehydrogenase; 1.2.3.14: abscisic-aldehyde oxidase; 2.4.1.263: abscisate beta-glucosyltransferase; 3.2.1.21: beta-glucosidase; 2.5.1.27: adenylate dimethylallyltransferase; 2.5.1.75: tRNA dimethylallyltransferase

The proteomic expression profile was consistent with the variation in hormones content of L. punctata after application of uniconazole. A previous study found that uniconazole regulates plant changes by inhibiting GA biosynthesis and ABA catabolism [42]. However, few studies have investigated the variation in these pathways in detail except for several reports on biochemical and physiological analyses [43]. There are C15 direct and C40 indirect pathways for ABA biosynthesis. Most ABA generates from $\mathrm{C} 40$ carotenoid through the indirect cleavage process in higher plants [44]. In our study, ABA in L. punctata is the most abundant hormone and its content increased to 2.7 times its original amount at $240 \mathrm{~h}$, which is approximately 14-fold more than ZR and GA. Proteomic analysis of the ABA biosynthetic pathway also showed that most of the enzymes, including GBA3, ZEP, ABA2, and especially the key enzyme NCED, had up-regulated expression. On the other hand, the GA and ZR content always had a relatively low value. Similarly, these enzymes involved in the biosynthetic pathways of GA and ZR, such as CPS, KS, G44OX, and GA30X, showed no differential change or were not detected. The transcripts encoding these enzymes also had a relatively low level of expression.

\section{Starch accumulation of $L$. punctata under uniconazole treatment}

The dry weight and starch content increased significantly after application of uniconazole. According to the calculated average, the dry weight of L. punctata in one flask increased continuously from 0.57 to $1.74 \mathrm{~g}$ by $240 \mathrm{~h}$ (Fig. 4). Meanwhile, there was a significant increase in starch content from 3.16 to $48.01 \%$ of DW by $240 \mathrm{~h}$. The dry weight and starch content increased 3.1and 15.2-fold, respectively. The activity of enzymes involved in starch biosynthesis and degradation was also analyzed. First, the activity of ADP-glucose pyrophosphorylase (EC: 2.7.7.27; AGP), the most important key enzyme in starch biosynthesis, increased from 8.20 to the maximum of $27.59 \mathrm{U} / \mathrm{mg}$ prot by $5 \mathrm{~h}$ (Fig. 4). Similarly, the activity of soluble starch synthetase (EC: 2.4.1.21; SSS), which promotes amylopectin biosynthesis, also increased from 8.03 to $39.29 \mathrm{U} / \mathrm{mg}$ prot by $5 \mathrm{~h}$. Furthermore, starch can be degraded by two amylases into dextrin and maltose to supply energy for utilization in life activities. The activities of alpha-amylase (EC: 3.2.1.1; $\alpha$-AMY) stabilized at a level of $0.003 \mathrm{U} / \mathrm{mg}$ prot. However, those of beta-amylase (EC: 3.2.1.2; $\beta$-AMY) increased from 0.032 to $0.345 \mathrm{U} / \mathrm{mg}$ prot by $240 \mathrm{~h}$.

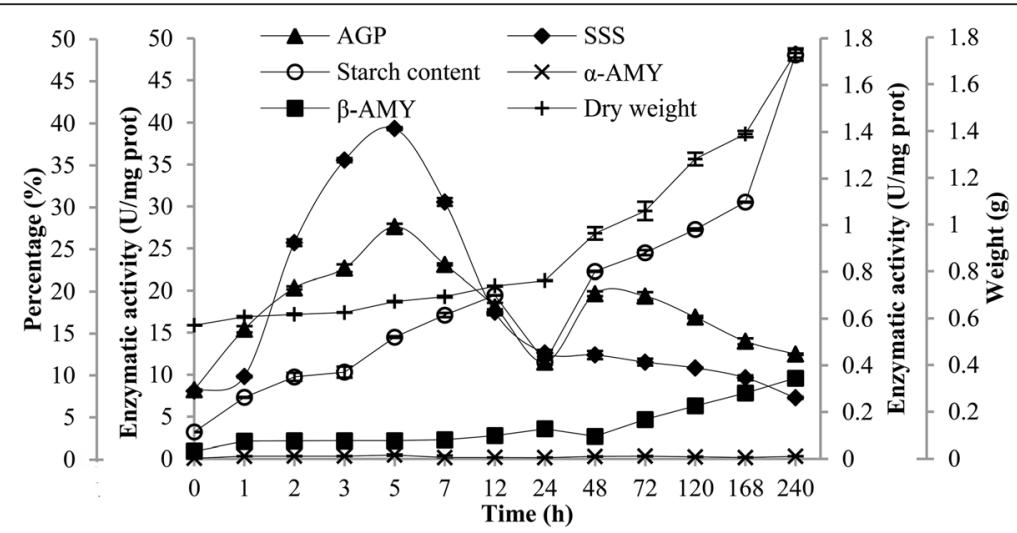

Fig. 4 Dry weight, starch content, and activities of AGP, SSS, $a$ - and $\beta$-AMY in duckweed. The starch content corresponds to the left of main Y-axis, activities of AGP and SSS correspond to the right of main $Y$-axis, activities of $a$ - and $\beta$-AMY correspond to the left of minor $Y$-axis, and dry weight corresponds to the right of minor $Y$-axis 
The expression profiles of enzymes involved in the starch metabolic pathway were analyzed further. To begin with, two key enzymes related to starch biosynthesis, AGP and granule bound starch synthase (EC: 2.4.1.242; GBSS), displayed up-regulated expression (Fig. 5). AGP, the first key enzyme responsible for ADPglucose synthesis and transfer, consists of two identical large subunits (AGP-LS) and two identical small subunits (AGP-SS). The AGP-LS and AGP-SS are in charge of regulatory function and catalytic activity, respectively [45]. GBSS, closely combined with starch granules, plays a key role in the biosynthesis of amylose. The results showed that AGP-LS and GBSS significantly increased 2.00 - and 5.29-fold at $240 \mathrm{~h}$, respectively. However, no significant increases were observed for other key enzymes or subunits, such as AGP-SS, soluble starch synthase (EC: 2.4.1.21; SSS) or starch branching enzyme (EC: 2.4.1.18; SBE). In addition, in the starch degradation process, $\alpha$-AMY and $\beta$-AMY play the chief roles in plants. $\alpha$-AMY displayed expression level decreases to 0.79 that of the control level at $5 \mathrm{~h}$, but $\beta$-AMY increased 1.34-fold at $240 \mathrm{~h}$. Finally, almost all enzymes involved in some other carbohydrate metabolic branches, which compete with starch biosynthesis for substrates to synthesize sucrose, cellulose and trehalose, shared no significant change or were unidentified. In sucrose biosynthesis, sucrose synthase (EC: 2.4.1.13; SuSy) displayed no significant change and sucrose-6-phosphate phosphatase (EC: 3.1.3.24; SPP) was not identified. However, sucrose-phosphate synthase (EC: 2.4.1.14; SPS) was identified and increased 1.97-fold at $240 \mathrm{~h}$. Cellulose synthase (EC: 2.4.1.12; CESAs), which catalyzes cellulose biosynthesis, was not identified. Neither trehalose-6phosphate synthase (EC: 2.4.1.15; TPS) nor trehalose-6phosphate phosphatase (EC: 3.1.3.12; TPP), which are involved in trehalose biosynthesis, displayed significant differences.

The proteomic analysis, enzymatic assay and composition determination were combined to understand the rapid high starch accumulation observed after application of uniconazole. The data were analyzed and compared at three different levels. Starch composition determination showed that starch content increased rapidly. At $240 \mathrm{~h}$, the starch percentage increased from 3.16 to $48.01 \%$ (DW) (Fig. 4). The mean of total starch weight in one flask increased from 1.8 to $83.5 \mathrm{mg}$, which meant that the starch quantity increased to 46.4 times its origin amount. Meanwhile, the enzymatic activity assay also indicated that activities of AGP and SSS, key enzymes related to starch biosynthesis, increased 3.4 and 4.9 times, respectively. Both $\alpha$-AMY and $\beta$-AMY had very low enzymatic activities at all samples, which were far below those of starch biosynthesis-related enzymes. Fig. 4 showed a dip in starch content and activities of AGP and SSS at $24 \mathrm{~h}$. In this research, there was $8 \mathrm{~h}$ dark from 12 to $20 \mathrm{~h}$, therefore, the dip may result from

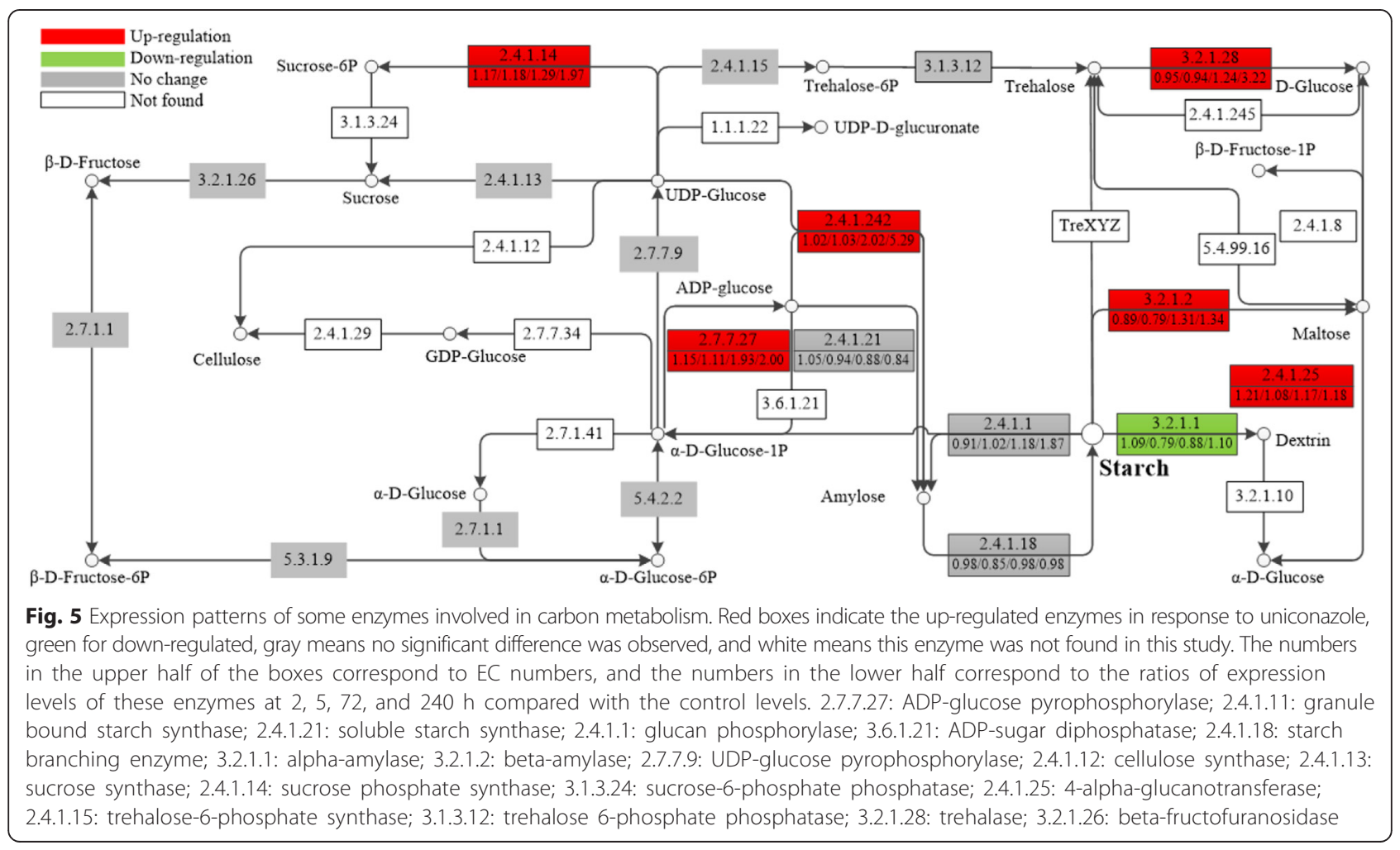


starch metabolism and lack of production in this period. Most importantly, proteomic data showed a very similar trend to the above mentioned results. On the one hand, uniconazole significantly up-regulated the expression levels of starch biosynthesis-related key enzymes, such as AGP-LS and GBSS (Fig. 5). On the other hand, the expression level of $\alpha$-AMY was slightly down-regulated, but that of $\beta$-AMY was slightly up-regulated. In addition, there was no significant difference in the expression levels of regulatory enzymes involved in other carbohydrate metabolic branches that compete for glucose, except for SPS, which was increased. We deduced that many enzymes were undetected due to a very low abundance under the detection limit. The starch biosynthesis-related enzymes were highly expressed and up-regulated, but the degradation-related enzymes were hardly expressed and down-regulated. These results further demonstrated that high starch accumulation may have predominantly resulted from the synergistic effect of regulated expression of these enzymes in the relevant pathways under uniconazole application, which were consistent with the transcriptomic data, enzymatic assay, and composition determination.

So far, few studies have been conducted that systematically investigate the uniconazole-induced ABA, GA, and ZR biosynthesis and starch accumulation in plants. Uniconazole is well known as a plant growth retardant through inhibition of GA biosynthesis [25]. The effects of uniconazole on GA biosynthesis and metabolism in rice plants have been well established [46]. Many previous researches about uniconazole mainly focus on its effects on plant height [47], improved grain quality [30], and enhancement of tolerance to stress [48]. This integrated study provided much information about endogenous hormone changes and hormone-regulated starch accumulation induced by the chemical growth regulator.

To understand the uniconazole-induced starch accumulation in L. punctata, a putative model was constructed, in which $\mathrm{ABA}$ and other endogenous hormones modulate starch accumulation in response to uniconazole application (Fig. 6). The significantly increased endogenous ABA induced by uniconazole may play the leading role in regulating starch accumulation instead of GA. According to the literature [42], uniconazole, as a potential inhibitor of ABA catabolism, also contributes to the enhancement of $\mathrm{ABA}$ content. As shown in the model, recent research found that early ABA signal transduction depends on pyrabactin resistance (PYR) protein and the regulatory component of the ABA receptor-type $2 \mathrm{C}$ protein phosphatases-snf1related protein kinase (PYR/RCAR-PP2C-SnRK2) pathway [49]. The PYRs are ABA receptors functioning at the apex of negative regulatory pathway that controls $A B A$ signaling. First, $A B A$ is bound by PYRs, which dissociate to form $\mathrm{ABA}$ receptor-PP2C complex to initiate $\mathrm{ABA}$ signaling pathway [50]. Therefore, complex formation inhibits the activity of the PP2C in an ABA-dependent manner, allowing activation of SnRK2s. Then, ABA-insensitive 4 (ABI4) is activated by phosphates of SnRK2, resulting in control of gene expression of APLs [51]. Finally, the transcription of $A P L s$ influences protein expression and enzymatic activity of ADP-glucose pyrophosphorylase (AGP). In this research, proteomic result showed that no significant difference was observed for the expression of PYR1 protein. AGP, a key enzyme in starch synthetic pathway, exhibited upregulated expression and increased enzymatic activity, resulting in starch accumulation. Wang [52] also gained high-starch dormant bud structure (turions) of S. polyrrihza in ABA enhanced medium, but it took a long time and could hardly be produced in full scale. After ABA treatment, the genes of APL2 and APL3 of S. polyrhiza were highly expressed in earlier stages of turions development. Moreover, GA induced release of potato tuber bud dormancy and expression of $\alpha$-amylase to mediate starch degradation [53]. Uniconazole-induced GA reduction may similarly induce low expression of $\alpha$-amylase, which also contributed to accumulate high levels of starch. Additionally, there may be an unknown regulated mechanism of starch accumulation, which is indicated by a question mark (Fig. 6). The change in endogenous hormone levels induced by uniconazole, including ABA, GA and others, coregulated the starch accumulation in L. punctata. This model interfaces with hormone change, signal transduction, protein expression, enzymatic activity, and starch accumulation, thus providing a mechanistic connection between the uniconazole application and starch accumulation.

\section{Conclusions}

In summary, uniconazole has the potential to enhance the quantity and quality of duckweed feedstock for biofuel production. A proteomic approach was employed to investigate the response of duckweed after application of uniconazole. The results demonstrated that rapid accumulation of high levels of starch resulted from regulated expression of enzymes in the relevant pathways, which were consistent with the transcriptomic data, enzymatic assay, and composition determination. This study not only provided insights into the molecular mechanisms of uniconazole-induced hormone variation and starch accumulation but also highlighted the potential for duckweed to be feedstock for biofuel as well as for sewage treatment.

\section{Methods}

Duckweed growth conditions and uniconazole application Landoltia punctata 0202, a wild duckweed, was collected from Sichuan province, China and stored in resource bank of duckweed in Chengdu Institute of Biology, Chinese Academy of Sciences. Ethics and standards of reporting in the research had been approved and consented from 


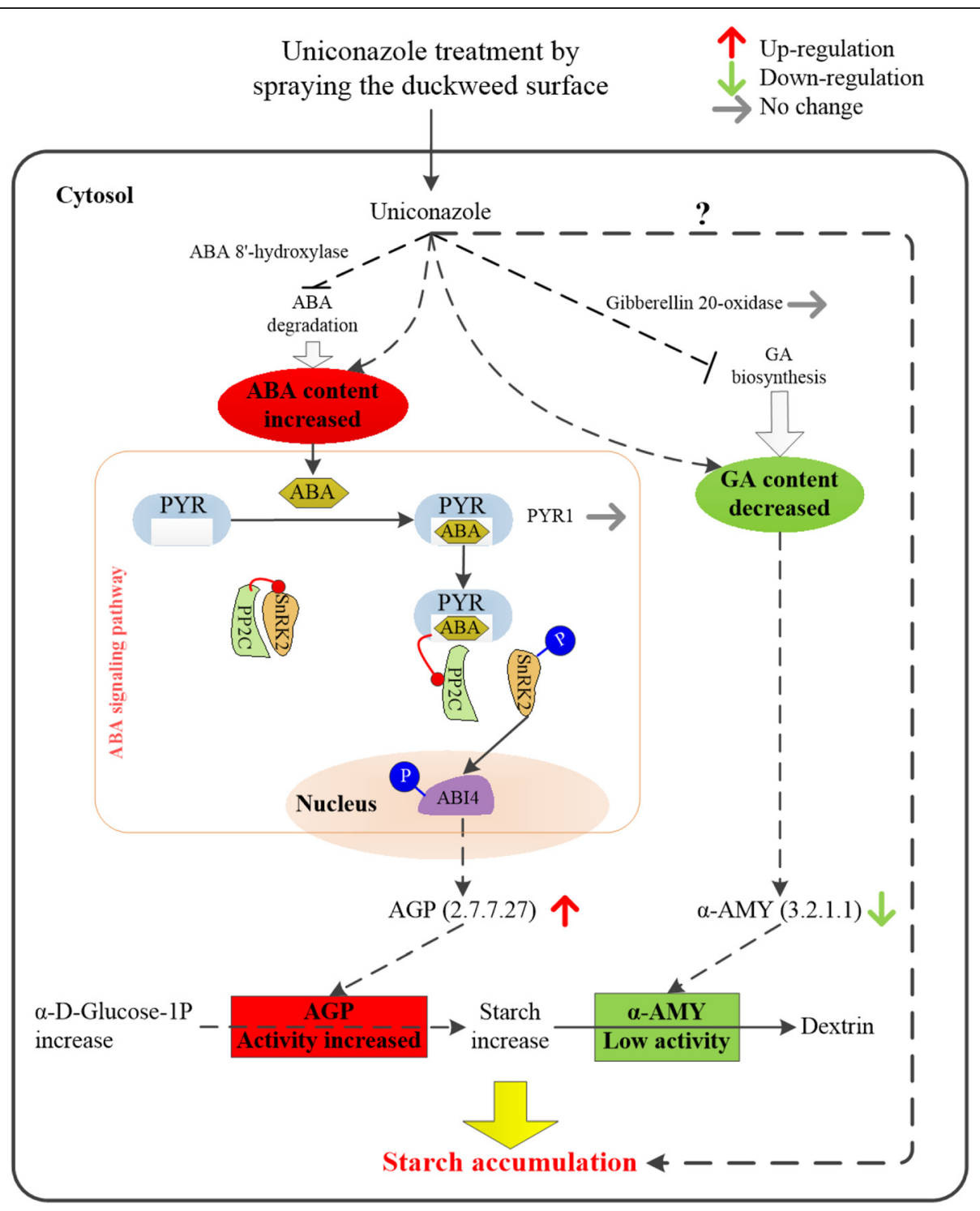

Fig. 6 A proposed model in which ABA and other endogenous hormones modulate starch accumulation. Red upward arrow indicates up-regulated protein in response to uniconazole, green downward arrow for down-regulated, gray arrow means no significant difference was observed in this research. The model was constructed to help understanding uniconazole-induced hormone variation and starch accumulation in $L$. punctata. Uniconazole induces in expression of hormone-associated enzymes that result in changes in endogenous hormone contents. ABA is bound by PYRs and then initiates ABA signaling pathway [49,50]. The significantly increased ABA and decreased GA modulate the expression of some enzymes involved in starch metabolism, and these enzymes finally result in starch accumulation. There may be an unknown regulation mechanism of starch accumulation, which is indicated by a question mark. See text for detailed description of the model. PYR: pyrabactin resistance; AGP: ADP-glucose pyrophosphorylase; a-AMY: alpha-amylase

researchers and participants. Experimental research on duckweed plants including collection and utilization of the material had complied with institutional, national, and international guidelines. Duckweed was cultivated in a standard 1/6 Hoagland nutrient solution ( $\mathrm{pH} 7.0)$ [54] in a commercial growth chamber (under a light intensity of $130 \mu \mathrm{mol}$ photons $\mathrm{m}^{-2} \mathrm{~s}^{-1}$, a photoperiod of $25{ }^{\circ} \mathrm{C}$ for $16 \mathrm{~h}$ a day and $15{ }^{\circ} \mathrm{C}$ for $8 \mathrm{~h}$ a night, $80 \%$ relative humidity). After 3 days, $6.0 \mathrm{~g}$ fresh weight of fronds were then transferred into a $1.0 \mathrm{~L}$ nutrient solution in a $1.0 \mathrm{~L}$ glass flask with uniconazole treatment over a period of 10 days under identical conditions. Uniconazole was dissolved in $10 \%$ methanol and then diluted with water. A total of $5 \mathrm{ml}$ aqueous solution containing $800 \mathrm{mg} / \mathrm{L}$ uniconazole (10\% methanol solution as a control) was applied as spraying the surface of a population after transferring. Uniconazole-P powder ( $\mathrm{S}: \mathrm{R}=79: 21$ ) was manufactured by Sumitomo Chemical (Osaka, Japan). After applying uniconazole, plants were harvested at a total of 13 different time points $(0,1,2,3,5,7,12,24,48,72,120,168$, 
and $240 \mathrm{~h}$ ). For each point, three biological replicates were carried out independently. Moreover, these samples were aliquoted, immediately snap-frozen in liquid nitrogen and stored at $-80{ }^{\circ} \mathrm{C}$ for proteomic analysis.

\section{Composition characterization and enzymatic activity assay}

A small number of the collected samples were parchdried at $70{ }^{\circ} \mathrm{C}$ for $48 \mathrm{~h}$ to determine the dry weight, as well as the starch content. The starch content was measured as glucose content in total sugar via HPLC (Thermo 2795, Thermo Corp, USA)-ELSD (All-Tech ELSD 2000, All-tech, Corp, USA), following the method of Zhang [4]. The starch content equals glucose content/1.1. The extraction, purification, and determination of endogenous levels of ABA, ZR, and GA using an indirect ELISA technique were performed as described by Wang [55] and Yang [56]. The activities of starch biosynthesis-related enzymes (AGP, SSS) and degradation-related enzymes ( $\alpha$-AMY, $\beta$-AMY) were measured using methods described by Nakamura and Hammond [57, 58].

\section{Protein extraction and digestion}

Determination of time points for proteomic analysis is vital to elucidate the rapid starch accumulation in response to uniconazole application. Five time points (0, 2, 5, 72, and $240 \mathrm{~h}$ ) were subjected to iTRAQ proteomic analysis according to the composition changes and enzymatic activity assay results.

For each sample, $0.5 \mathrm{~g}$ fronds was ground into a powder in liquid nitrogen and resuspended in lysis buffer (8 M urea, 4 \% 3-[(3-cholamidopropyl) dimethylammonio]-1-propanesulfonate (CHAPS), $40 \mathrm{mM}$ Tris- $\mathrm{HCl}$ ) with $1 \mathrm{mM}$ phenylmethanesulfonyl fluoride (PMSF) and $2 \mathrm{mM}$ ethylenediaminetetraacetic acid (EDTA) at a ratio 1:5 (W/V). After 5 min of vigorous vortexing, dithiothreitol (DTT) was also added to make a final concentration of $10 \mathrm{mM}$. The cell mixture was subjected to sonication for $15 \mathrm{~min}$ and placed at ambient temperature for $30 \mathrm{~min}$. The mixture was centrifuged for $60 \mathrm{~min}$ at $40,000 \times g$ at $10{ }^{\circ} \mathrm{C}$, and the supernatant was mixed well with $10 \%$ pre-cooled TCA/acetone $(1: 4, \mathrm{v} / \mathrm{v})$, followed by precipitation at $-20{ }^{\circ} \mathrm{C}$ overnight. The samples were then centrifuged for $60 \mathrm{~min}$ at $40,000 \times g$ at $10{ }^{\circ} \mathrm{C}$. After the cell pellets were washed twice with cold acetone and dried, they were dissolved in digestion buffer $(500 \mathrm{mM}$ triethylammonium bicarbonate TEAB, $0.05 \% \mathrm{w} / \mathrm{v}$ sodium dodecyl sulfate, SDS), aliquoted and stored at $-80{ }^{\circ} \mathrm{C}$. The protein concentration was determined using the Bradford method. After quantifying the concentration, equal aliquots $(100 \mu \mathrm{g})$ from each sample were set up in a centrifuge tube and digested by incubation at $37{ }^{\circ} \mathrm{C}$ overnight with trypsin (Sigma; 1:20 w/w added at 0 and $4 \mathrm{~h}$ ) and lyophilized.
iTRAQ labeling, SCX fractionation and LC-ESI-MS/MS analysis The iTRAQ labeling of digested peptide samples was performed following the manufacturer's protocol with the iTRAQ ${ }^{\circ}$ Reagent 8-plex Kit (AB SCIEX, USA). Five samples $(0,2,5,72$, and $240 \mathrm{~h})$ were labeled with iTRAQ tags 114, 115, 117, 119, and 121, respectively. The labeled peptides were then combined and vacuum centrifuged to dryness. After combining the four labeled samples, they were fractionated using strong cation exchange chromatography. The iTRAQ labeled peptides were dissolved in $4 \mathrm{~mL}$ of buffer solvent A $(25 \mathrm{mM}$ $\mathrm{NaH}_{2} \mathrm{PO}_{4}, 25 \%$ acetonitrile, $\mathrm{pH}$ 2.7), centrifuged for $20 \mathrm{~min}$ at $14,000 \times g$ and loaded onto a Ultremex SCX column $(250 \times 4.6 \mathrm{~mm}, 5 \mu \mathrm{m})$ using an Agilent series 1100 HPLC instrument (Agilent technologies) at a flow rate of $1.0 \mathrm{~mL} / \mathrm{min}$. The column was first equilibrated in $95 \%$ solvent A (mentioned above) for $10 \mathrm{~min}$, and the following gradient elution program was then used: $5 \%$ solvent B $\left(25 \mathrm{mM} \mathrm{NaH}_{2} \mathrm{PO}_{4}, 1 \mathrm{M} \mathrm{KCl}\right.$ in $25 \%$ acetonitrile, $\mathrm{pH} \mathrm{2.7)} \mathrm{for} 7 \mathrm{~min}, 5-60 \% \mathrm{~B}$ for $20 \mathrm{~min}, 60-$ $100 \% \mathrm{~B}$ for $2 \mathrm{~min}$, and $100 \% \mathrm{~B}$ for $1 \mathrm{~min}$. A total of 20 fractions were collected based on the ultraviolet absorption with $214 \mathrm{~nm}$. The collected fractions were desalted with StrataX column and concentrated to dryness using a vacuum centrifuge for LC-ESI-MS/MS analysis.

The LC-MS/MS analysis was performed using an $A B$ SCIEX TripleTOF ${ }^{\mathrm{Tm}} 5600$ mass spectrometer (AB SCIEX, USA) coupled with a Tempo Nano HPLC system (AB SCIEX, USA). Each fraction was reconstituted in buffer A (5 \% acetonitrile $0.1 \%$, formic acid) with a concentration of $0.5 \mu \mathrm{g} / \mu \mathrm{l}$ and then centrifuged for $10 \mathrm{~min}$ at $12,000 \times g$ to obtain the supernatant. The fractioned peptides were separated with a nanobored C18 column with a picofrit nanospray tip $(75 \mu \mathrm{m}$ ID $\times 150 \mathrm{~mm}, 5 \mu \mathrm{m}$ particles; New Objective, USA). Then, $5 \mu \mathrm{L}$ fraction solutions were loaded onto the LC-Nano ESI-MS/MS system at a flow rate of $8 \mu \mathrm{l} / \mathrm{min}$ for $4 \mathrm{~min}$ with a splitter to obtain an effective flow rate of $0.3 \mu \mathrm{l} / \mathrm{min}$. The peptides were eluted using the following procedure: $5 \%$ solvent B (95\% acetonitrile, and $0.1 \%$ formic acid) for $5 \mathrm{~min}, 5-$ $35 \%$ B for $35 \mathrm{~min}, 35-60 \%$ B for $5 \mathrm{~min}, 60-80 \%$ B for $2 \mathrm{~min}, 80 \% \mathrm{~B}$ for $2 \mathrm{~min}, 80-5 \% \mathrm{~B}$ for $1 \mathrm{~min}$ and $5 \% \mathrm{~B}$ for $2 \mathrm{~min}$. Peptides were electro-sprayed into the orifice of the mass spectrometer through coated silica tips at a high ion spray voltage of $2.5 \mathrm{kV}$. For MS data acquisition, the mass spectrometer was set to positive ion mode with a selected mass range $350-1250 \mathrm{~m} / \mathrm{z}$ and TOF-MS accumulation time $0.25 \mathrm{~s}$ to detect precursor ions. Peptide ions with charge states $2^{+}$to $4^{+}$were selected for MS/MS. Information dependent acquisition of MS/MS was performed on the three most abundant peptides exceeding five counts were selected for MS/MS, and dynamic exclusion time was set at $30 \mathrm{~s}$ with $\pm 30 \mathrm{mDa}$ mass tolerance. 


\section{Proteomic data analysis}

The MS/MS data acquisition was performed with Analyst ${ }^{\oplus}$ QS 2.0 software (AB SCIEX). Raw data files acquired from the orbitrap were converted into MGF files. For protein identification, the data were processed by thoroughly searching against a database generated from our transcriptome data of L. punctata under uniconazole treatment using Mascot 2.3.02 (Matrix Science, London, United Kingdom) [59]. The transcriptome data were deposited in NCBI's Transcriptome Shotgun Assembly (TSA) database under the accession number of PRJNA242298. Of the 140,432 contigs, 91,303 (65.0\%) got annotation information. For protein identification, the labeled peptides were automatically selected by the algorithm to calculate the reporter peak area, error factor and $p$-value. The relative abundances were determined using the reporter ion peak areas [60]. The parameters in the software were as follows: max missed cleavages, 1; enzyme, trypsin; cysteine alkylation, MMTS; species, plant; detected protein threshold, $\pm 0.05 \mathrm{Da}$; peptide mass tolerance, $20 \mathrm{ppm}$; FDR determination of protein at $1 \%$ FDR and distinct peptides at $5 \%$. Proteins with a fold change $>1.2$ or $<0.8$ among different comparisons with a $p$-value less than 0.05 were considered to be differentially expressed proteins. Functional annotation of the proteins identified was performed using Blast2GO software, and GO categorization was achieved using WEGO [http://wego.genomics.org.cn/ cgi-bin/wego/index.pl]. The KEGG pathway enrichment analysis of the responsive proteins was conducted according to the formula [61] from the KEGG Pathway Database [http://www.genome.jp/kegg/].

\section{Availability of supporting data}

The transcriptome data set supporting the results of this article is available in the [NCBI's Transcriptome Shotgun Assembly (TSA) database] repository, under the accession number of PRJNA242298 [unique persistent identifier and hyperlink to dataset in http://www.ncbi.nlm.nih.gov/gquery/?term=PRJNA242298]. The data set supporting the results of this article is included within the article.

\section{Additional files}

Additional file 1: Up-regulated proteins after application of uniconazole. The up-regulated proteins with $>1.2$-fold change and $p$-value less than 0.05 and their ratios among all comparative groups listed in the table. (XLSX 64 kb)

Additional file 2: Down-regulated proteins after application of uniconazole. The down-regulated proteins with $<0.8$-fold change and $p$-value less than 0.05 and their ratios among all comparative groups listed in the table. (XLSX $46 \mathrm{~kb}$ )

Additional file 3: KEGG pathway analysis of the annotated proteins. The pathway, number of annotated proteins, and pathway ID were listed in the table. (XLSX $15 \mathrm{~kb}$ )

\section{Abbreviations}

DW: Dry weight; iTRAQ: Isobaric tags for relative and absolute quantification; LC-MS/MS: Liquid chromatography-tandem mass spectrometry; EC: Enzyme codes; GO: Gene Ontology; KEGG: Kyoto Encyclopedia of Genes and Genomes; ABA: Abscisic acid; CKs: Cytokinins; GAs: Gibberellins.

\section{Competing interests}

The authors declare that they have no competing interests.

\section{Authors' contributions}

$\mathrm{HZ}, \mathrm{KH}, \mathrm{XM}, \mathrm{YF}$, and $\mathrm{YJ}$ conceived of the study. MH and $\mathrm{YF}$ drafted the manuscript. $\mathrm{HZ}, \mathrm{KH}, \mathrm{XM}$ and $\mathrm{YJ}$ revised the manuscript. $\mathrm{MH}, \mathrm{YF}, \mathrm{YL}$, JS and $X T$ conducted the experiments and proteomic analysis. All authors read and approved the final manuscript.

\section{Acknowledgements}

The research was supported by the National Key Technology R\&D Program of China (No. 2015BAD15B01), the Projects of International Cooperation of Ministry of Science and Technology of China (No. 2014DFA30680), West Light Foundation of The Chinese Academy of Sciences (No. Y2C5021100), and Key Laboratory of Environmental and Applied Microbiology, Chengdu Institute of Biology, Chinese Academy of Sciences (No. KLCAS-2014-02). We would also like to thank Dr. Weizao Huang for revising the manuscript.

\section{Author details}

${ }^{1}$ Chengdu Institute of Biology, Chinese Academy of Sciences, Chengdu 610041, China. ${ }^{2}$ University of Chinese Academy of Sciences, Beijing 100049, China. ${ }^{3}$ Key Laboratory of Environmental and Applied Microbiology, Chinese Academy of Sciences, Chengdu 610041, China. ${ }^{4}$ Environmental Microbiology Key Laboratory of Sichuan Province, Chengdu 610041, China.

Received: 25 March 2015 Accepted: 29 August 2015

Published online: 15 September 2015

\section{References}

1. Goldemberg J. Ethanol for a sustainable energy future. Science. 2007;315(5813):808-10.

2. Quintero J, Montoya M, Sánchez O, Giraldo O, Cardona C. Fuel ethanol production from sugarcane and corn: comparative analysis for a Colombian case. Energy. 2008;33(3):385-99.

3. Papong S, Malakul P. Life-cycle energy and environmental analysis of bioethanol production from cassava in Thailand. Bioresour Technol. 2010;101:S112-8.

4. Zhang LA, Chen QA, Jin YL, Xue HL, Guan JF, Wang ZY, et al. Energy-saving direct ethanol production from viscosity reduction mash of sweet potato at very high gravity (VHG). Fuel Process Technol. 2010;91(12):1845-50.

5. Simmons BA, Loque D, Blanch HW. Next-generation biomass feedstocks for biofuel production. Genome Biol. 2008:9(12):242-8.

6. Field CB, Campbell JE, Lobell DB. Biomass energy: the scale of the potential resource. Trends Ecol Evol. 2008;23(2):65-72.

7. Li Q, Song J, Peng S, Wang JP, Qu GZ, Sederoff RR, et al. Plant biotechnology for lignocellulosic biofuel production. Plant Biotechnol J. 2014;12(9):1174-92.

8. Tam SM, Boyce PC, Upson TM, Barabe D, Bruneau A, Forest F, et al. Intergeneric and infrafamilial phylogeny of subfamily Monsteroideae (Araceae) revealed by chloroplast trnL-F sequences. Am J Bot. 2004;91(3):490-8.

9. Porath D, Hepher B, Koton A. Duckweed as an aquatic crop: Evaluation of clones for aquaculture adherence. Aquat Bot. 1979;7(3):273-8.

10. Xiao $Y$, Fang $Y$, Jin $Y L$, Zhang GH, Zhao H. Culturing duckweed in the field for starch accumulation. Ind Crops Prod. 2013:48(2013):183-90.

11. Chen $Q$, Jin $Y L$, Zhang GH, Fang Y, Xiao Y, Zhao H. Improving Production of Bioethanol from Duckweed (Landoltia punctata) by Pectinase Pretreatment. Energies. 2012;5(8):3019-32.

12. $\mathrm{Xu} \mathrm{JL,} \mathrm{Cui} \mathrm{WH,} \mathrm{Cheng} \mathrm{JJ,} \mathrm{Stomp} \mathrm{AM.} \mathrm{Production} \mathrm{of} \mathrm{high-starch} \mathrm{duckweed}$ and its conversion to bioethanol. Biosyst Eng. 2011;110(2):67-72.

13. Stomp AM. The duckweeds: a valuable plant for biomanufacturing. Biotechnol Annu Rev. 2005;11:69-99.

14. Su HF, Zhao Y, Jiang J, Lu QL, Li Q, Luo Y, et al. Use of Duckweed (Landoltia punctata) as a Fermentation Substrate for the Production of Higher Alcohols as Biofuels. Energy Fuel. 2014;28(5):3206-16. 
15. Ge XM, Zhang NN, Phillips GC, Xu JF. Growing Lemna minor in agricultural wastewater and converting the duckweed biomass to ethanol. Bioresour Technol. 2012;124:485-8.

16. Bayrakci AG, Kocar $G$. Second-generation bioethanol production from water hyacinth and duckweed in Izmir: A case study. Renew Sust Energ Rev. 2014;30:306-16.

17. Muradov N, Taha M, Miranda AF, Kadali K, Gujar A, Rochfort S, et al. Dual application of duckweed and azolla plants for wastewater treatment and renewable fuels and petrochemicals production. Biotechnol Biofuels 2014;7(1):30-47.

18. Cheng JJ, Stomp AM. Growing duckweed to recover nutrients from wastewaters and for production of fuel ethanol and animal feed. Clean-Soil Air Water. 2009;37(1):17-26.

19. Oron G. Duckweed culture for wastewater renovation and biomass production. Agr Water Manage. 1994;26(1):27-40.

20. Zhao $Y$, Fang $Y$, Jin $Y$, Huang J, Bao S, Fu T, et al. Potential of duckweed in the conversion of wastewater nutrients to valuable biomass: A pilot-scale comparison with water hyacinth. Bioresour Technol. 2014;163(2014):82-91.

21. Cui W, Xu J, Cheng JJ, Stomp AM. Starch accumulation in duckweed for bioethanol production. Biological Engineering Transactions. 2011;3(4):187-97.

22. Yin $Y, Y u C, Y u$, Z Zhao J, Sun C, Ma Y, et al. The influence of light intensity and photoperiod on duckweed biomass and starch accumulation for bioethanol production. Bioresour Technol. 2015;187(2015):84-90.

23. Zhao H, Appenroth $K$, Landesman L, Salmean AA, Lam E. Duckweed rising at Chengdu: summary of the 1st International Conference on Duckweed Application and Research. Plant Mol Biol. 2012;78(6):627-32.

24. Lam E, Appenroth KJ, Michael T, Mori K, Fakhoorian T. Duckweed in bloom the 2nd International Conference on Duckweed Research and Applications heralds the return of a plant model for plant biology. Plant Mol Biol. 2014;84(6):737-42.

25. Davis TD, Curry EA, Steffens GL. Chemical regulation of vegetative growth Crit Rev Plant Sci. 1991;10(2):151-88

26. Jaleel CA, Kishorekumar A, Manivannan P, Sankar B, Gomathinayagam M, Gopi R, et al. Alterations in carbohydrate metabolism and enhancement in tuber production in white yam (Dioscorea rotundata Poir.) under triadimefon and hexaconazole applications. Plant Growth Regul. 2007:53(1):7-16.

27. Duan L, Guan C, Li J, Eneji AE, Li Z, Zhai Z. Compensative effects of chemical regulation with uniconazole on physiological damages caused by water deficiency during the grain filling stage of wheat. J Agron Crop Sci. 2008;194(1):9-14

28. Leul M, Zhou WJ. Alleviation of waterlogging damage in winter rape by application of uniconazole - Effects on morphological characteristics, hormones and photosynthesis. Field Crop Res. 1998;59(2):121-7.

29. Yang W-y, Z-f X, W-j R, Wang X-c. Effect of S-3307 on nitrogen metabolism and grain protein content in rice. Zhongguo Shuidao Kexue. 2005;19(1):63-7.

30. Han $\mathrm{H}$, Yang $\mathrm{W}$. Influence of uniconazole and plant density on nitrogen content and grain quality in winter wheat in South China. Plant Soil Environ. 2009;55(4):159-66.

31. Zhan $\mathrm{G}$. Effects of plant growth regulators on contents of reduced sugar and starch in solanum tuberosum L. J Anhui Agi Sci. 2011;11:68-72.

32. Masuda J, Ozaki Y, Okubo H. Regulation in rhizome transition to storage organ in lotus (Nelumbo nucifera Gaertn.) with exogenous gibberellin, gibberellin biosynthesis inhibitors or abscisic acid. J Jpn Soc Hortic Sci. 2012;81(1):67-71

33. Huang $M$, Fang $Y$, Xiao $Y$, Sun J, Jin $Y$, Tao $X$, et al. Proteomic analysis to investigate the high starch accumulation of duckweed (Landoltia punctata) under nutrient starvation. Ind Crops Prod. 2014:59(2014):299-308.

34. Wang HX, Alvarez S, Hicks LM. Comprehensive comparison of iTRAQ and label-free LC-based quantitative proteomics approaches using two Chlamydomonas reinhardtii strains of interest for biofuels engineering J Proteome Res. 2012;11(1):487-501.

35. Ning K, Nesvizhskii Al. The utility of mass spectrometry-based proteomic data for validation of novel alternative splice forms reconstructed from RNA-Seq data: a preliminary assessment. BMC Bioinformatics. 2010;11:14-20.

36. Wang H, Zhang H, Wong YH, Voolstra C, Ravasi T, B Bajic V, et al. Rapid transcriptome and proteome profiling of a non-model marine invertebrate, Bugula neritina. Proteomics. 2010;10(16):2972-81.
37. Zeng J, Liu Y, Liu W, Liu X, Liu F, Huang P, et al. Integration of transcriptome, proteome and metabolism data reveals the alkaloids biosynthesis in Macleaya cordata and Macleaya microcarpa. PLoS One. 2013:8(1):e53409.

38. Huang SQ, Chen L, Te RG, Qiao JJ, Wang JX, Zhang WW. Complementary iTRAQ proteomics and RNA-seq transcriptomics reveal multiple levels of regulation in response to nitrogen starvation in Synechocystis sp PCC 6803 Mol Biosyst. 2013;9(10):2565-74.

39. Tao X, Fang Y, Xiao Y, Jin YL, Ma XR, Zhao Y, et al. Comparative transcriptome analysis to investigate the high starch accumulation of duckweed (Landoltia punctata) under nutrient starvation. Biotechnol Biofuels. 2013;6(1):72-89.

40. Lefebvre V, North H, Frey A, Sotta B, Seo M, Okamoto M, et al. Functional analysis of Arabidopsis NCED6 and NCED9 genes indicates that ABA synthesized in the endosperm is involved in the induction of seed dormancy. Plant J. 2006;45(3):309-19.

41. Lee $\mathrm{KH}$, Piao HL, Kim H-Y, Choi SM, Jiang F, Hartung W, et al. Activation of glucosidase via stress-induced polymerization rapidly increases active pools of abscisic acid. Cell. 2006;126(6):1109-20.

42. Saito S, Okamoto M, Okamoto M, Kushiro T, Koshiba T, Kamiya Y, et al. A plant growth retardant, uniconazole, is a potent inhibitor of $A B A$ catabolism in Arabidopsis. Biosci Biotech Bioch. 2006;70(7):1731-9.

43. Yan $W$, Yanhong $Y$, Wenyu $Y$, Taiwen $Y$, Weiguo $L$, Xiaochun W. Responses of root growth and nitrogen transfer metabolism to uniconazole, a growth retardant, during the seedling stage of soybean under relay strip intercropping system. Commun Soil Sci Plant Anal. 2013;44(22):3267-80.

44. Cowan AK, Richardson GR. Carotenogenic and abscisic acid biosynthesizing activity in a cell-free system. Physiol Plant. 1997;99(3):371-8.

45. Hannah LC, Shaw JR, Giroux MJ, Reyss A, Prioul JL, Bae JM, et al. Maize genes encoding the small subunit of ADP-glucose pyrophosphorylase. Plant Physiol. 2001;127(1):173-83.

46. Izumi K, Nakagawa S, Kobayashi M, Oshio H, Sakurai A, Takahashi N. Levels of laa, Cytokinins, Aba and ethylene in rice plants as affected by a gibberellin biosynthesis inhibitor, uniconazole-P. Plant Cell Physiol. 1988;29(1):97-104.

47. Schluttenhofer CM, Massa GD, Mitchell CA. Use of uniconazole to contro plant height for an industrial/pharmaceutical maize platform. Ind Crops Prod. 2011;33(3):720-6.

48. Zhang MC, Duan LS, Tian XL, He ZP, Li JM, Wang BM, et al. Uniconazoleinduced tolerance of soybean to water deficit stress in relation to changes in photosynthesis, hormones and antioxidant system. J Plant Physiol. 2007;164(6):709-17.

49. Hubbard KE, Nishimura N, Hitomi K, Getzoff ED, Schroeder Jl. Early abscisic acid signal transduction mechanisms: newly discovered components and newly emerging questions. Gene Dev. 2010;24(16):1695-708.

50. Park SY, Fung P, Nishimura N, Jensen DR, Fujii H, Zhao Y, et al. Abscisic acid inhibits type $2 \mathrm{C}$ protein phosphatases via the PYR/PYL family of START proteins. Science. 2009;324(5930):1068-71.

51. Akihiro T, Mizuno K, Fujimura T. Gene expression of ADP-glucose pyrophosphorylase and starch contents in rice cultured cells are cooperatively regulated by sucrose and ABA. Plant Cell Physiol. 2005;46(6):937-46

52. Wang WQ, Messing J. Analysis of ADP-glucose pyrophosphorylase expression during turion formation induced by abscisic acid in Spirodela polyrhiza (greater duckweed). Bmc Plant Biol. 2012;12:5-19.

53. Rentzsch S, Podzimska D, Voegele A, Imbeck M, Muller K, Linkies A, et al. Dose- and tissue-specific interaction of monoterpenes with the gibberellinmediated release of potato tuber bud dormancy, sprout growth and induction of alpha-amylases and beta-amylases. Planta. 2012;235(1):137-51.

54. Hoagland DR, Arnon DI. The water-culture method for growing plants without soil. Circular California Agricultural Experiment Station. 1950;347.

55. Wang Y, Li B, Du MW, Eneji AE, Wang BM, Duan LS, et al. Mechanism of phytohormone involvement in feedback regulation of cotton leaf senescence induced by potassium deficiency. J Exp Bot. 2012;63(16):5887-901.

56. Yang YM, Xu CN, Wang BM, Jia JZ. Effects of plant growth regulators on secondary wall thickening of cotton fibres. Plant Growth Regul. 2001;35(3):233-7.

57. Nakamura Y, Yuki K, Park S-Y, Ohya T. Carbohydrate metabolism in the developing endosperm of rice grains. Plant Cell Physiol. 1989;30(6):833-9.

58. Hammond JBW, Burton KS. Leaf starch metabolism during the growth of pepper (Capsicum annuum) plants. Plant Physiol. 1983;73(1):61-5. 
59. Charbonneau ME, Girard V, Nikolakakis A, Campos M, Berthiaume F, Dumas $F$, et al. O-linked glycosylation ensures the normal conformation of the autotransporter adhesin involved in diffuse adherence. J Bacteriol. 2007;189(24):8880-9.

60. Ford KL, Cassin A, Bacic A. Quantitative proteomic analysis of wheat cultivars with differing drought stress tolerance. Front Plant Sci. 2011;2:44-55.

61. Liu J, Chen L, Wang J, Qiao J, Zhang W. Proteomic analysis reveals resistance mechanism against biofuel hexane in Synechocystis sp. PCC 6803. Biotechnol Biofuels. 2012;5(1):68-85.

Submit your next manuscript to BioMed Central and take full advantage of:

- Convenient online submission

- Thorough peer review

- No space constraints or color figure charges

- Immediate publication on acceptance

- Inclusion in PubMed, CAS, Scopus and Google Scholar

- Research which is freely available for redistribution 